Yair Rosenthal at Rutgers University in New Brunswick, New Jersey, and his colleagues analysed sediment cores in Indonesia's Makassar

either crash into the spiral or hover in front of it.

This simple method of adjusting speed for landings could be widespread among animals and might even be useful in flying robots in the future, the authors suggest.

Proc. Natl Acad. Sci. USA http://doi.org/psp (2013)

\section{GENETICS}

\section{More risk genes for Alzheimer's}

The largest genetics study so far of late-onset Alzheimer's disease has identified 11 new genome regions that alter the risk of the disease.

A team of some 200 scientists in the International Genomics of Alzheimer's Project carried out meta-analyses of four separate genomewide association studies, profiling 17,008 people with Alzheimer's and 37,154 people who did not have the disease. The researchers reproduced their findings by genotyping an independent group of 8,572 Alzheimer's cases and 11,312 controls.

Their results confirmed nine out of ten known Alzheimer's risk genes. Eleven newly identified regions may give more clues about the biology of the disease, for which there are still no effective treatments.

Nature Genet. http://doi.org/ ppm (2013)

\section{PALAEOCLIMATOLOGY}

\section{Deep ocean is a heat sink}

A long-term reconstruction of temperatures in the Pacific Ocean reveals how its heat content has changed since the last glacial era - findings that could augment climate models.
Strait, and used the mineral

composition of tiny sea creatures' shells to calculate past water temperatures.

This showed that, at depths of 500-900 metres, temperatures in the Pacific Ocean were typically higher over the past several thousand years than at present. Over millennia, the waters have undergone prolonged cooling and temperatures are now increasing faster than at any time in the past 10,000 years.

The results support other studies suggesting that the ocean is currently taking up more heat, temporarily slowing the rise in average global air temperatures. Science 342, 617-621 (2013)

\section{ASTRONOMY}

\section{Explosions in the young Universe}

The biggest ever thermonuclear blasts happened in the early Universe, when primordial gas clumps collapsed and created the seeds of supermassive black holes.

Arising even before many stars and galaxies had time to form, the origins of big black holes have been a puzzle. Daniel Whalen of Los Alamos National Laboratory in New Mexico and his colleagues used computer simulations to reveal how gas in early galaxies could have cooled rapidly, forming stars that then exploded. The stars quickly collapsed back on themselves, generating turbulence that caused nearby gas clumps to form baby black holes.

In related work, the same team ran other analyses predicting whether light from those supernova blasts would be visible to upcoming space missions. The James Webb Space Telescope, due

COMMUNITY CHOICE

\title{
Cells grow in sleeping brains
}

\section{HIGHLY READ \\ on jneurosci.org in October}

Sleep seems to wake up a type of brain cell that maintains healthy neuron signalling. Chiara Cirelli and her colleagues at the

University of Wisconsin-Madison analysed support cells called oligodendrocytes that wrap around neurons and protect them with a specialized sheath that helps their signals to travel faster.

They collected oligodendrocytes and their precursor cells from mice after the animals had experienced a few hours of sleep or wakefulness and then profiled the expression of newly transcribed genes. Genes that were most active during wakefulness were involved in cell differentiation and death. By contrast, genes that were expressed during sleep promoted cell growth and the production of protective sheaths.

Further experiments showed that the rate of growth of oligodendrocyte precursor cells doubles during sleep, and that cell production increased when mice spent more time in the stage of sleep associated with dreaming.

J. Neurosci. 33, 14288-14300 (2013)

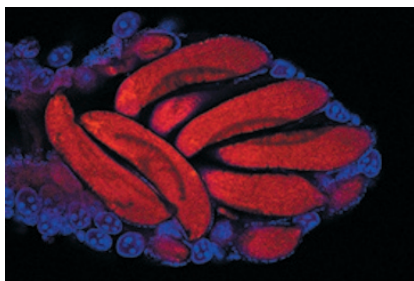

for launch in 2018, should be able to detect these stellar explosions, the authors find. Astrophys. J. 777, 99; 110 (2013)

\section{ENTOMOLOGY \\ Male mosquito boosts egg-making}

Male mosquitos deliver more than sperm when they copulate with females: they also provide a hormone that spurs egg development.

A team led by Flaminia Catteruccia at the Harvard School of Public Health in Boston, Massachusetts, studied the malaria-spreading mosquito Anopheles gambiae, focusing on a protein that is more abundant in females after mating. Tests using mosquito ovaries showed that this protein helps to direct nutrients into developing eggs (pictured,

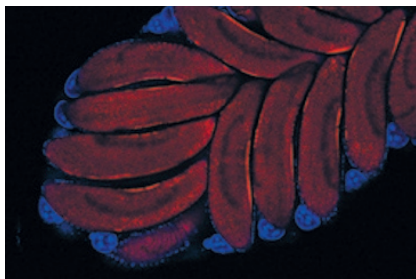

red shapes): when the protein was silenced, fewer eggs were produced (left) than when it was expressed (right).

Further work showed that a molecule in male mating secretions binds to this female protein and boosts its expression, which in turn increases egg production.

This is the first demonstration in insects of an interaction between a male hormone and a female protein that changes female reproductive success, the authors say. Interfering with it, perhaps by creating males that lack the hormone, might help to control populations of disease-carrying mosquitoes. PLoS Biol. 11, e1001695 (2013)

\section{DNATURE.COM}

For the latest research published by Naturevisit:

www.nature.com/latestresearch 